\title{
On products of starlike functions. I
}

\author{
by Georgi Dimkov (Sofia)
}

\begin{abstract}
We deal with functions given by the formula $F(z)=z G^{\prime}(z)=$ $z \prod_{j=1}^{n}\left(f_{j}(z) / z\right)^{a_{j}}$ where $f_{j}(z)$ are starlike of order $\alpha_{j}$ and $a_{j}$ are complex constants. In particular, radii of starlikeness and convexity as well as orders of starlikeness and convexity are found.
\end{abstract}

Introduction. Let $S$ be a class of all normalized functions $f(z)=z+\ldots$ that are analytic and univalent in the unit disc. We shall also use the following well known notations:

$$
S^{*}(\alpha)=\left\{f \in S: \operatorname{Re} \frac{z f^{\prime}(z)}{f(z)}>\alpha,|z|<1\right\}
$$

for the functions starlike of order $\alpha$ in the unit disc, and

$$
K(\alpha)=\left\{f \in S: \operatorname{Re}\left(1+\frac{\left.z f^{\prime \prime} z\right)}{f^{\prime}(z)}\right)>\alpha,|z|<1\right\}
$$

for the functions convex of order $\alpha$ in the unit disc, where $\alpha \in[0,1)$.

Silverman [1] obtained the order of starlikeness of functions given by

$$
z \prod_{j=1}^{m}(f(z) / z)^{a_{i}} \prod_{j=1}^{n}\left(g^{\prime}(z)\right)^{b_{j}}
$$

where $f \in S^{*}(\alpha), g \in K(\beta)$ and $a_{i}, b_{j} \geq 0$, as well as some other results.

Our aim is to generalize and make more precise the investigations of Silverman.

Starlikeness and convexity. We begin with the following remark. By the theorem Alexander [2] the class of functions of the kind $f(z) / z$ where $f \in S^{*}(\alpha)$ coincides with the class of the derivatives of the functions in $K(\alpha)$. This allows us to investigate, instead of the above-mentioned

1991 Mathematics Subject Classification: Primary 30C75. 
"double" product, either the product $\prod_{j=1}^{n}\left(f_{j}(z) / z\right)^{a_{j}}$ or $\prod_{j=1}^{n}\left(g_{j}^{\prime}(z)\right)^{b_{j}}$. We choose the first one.

Let $f_{j} \in S^{*}\left(\alpha_{j}\right), 0 \leq \alpha_{j} \leq 1$ and $a_{j}=\left|a_{j}\right| e^{i \theta_{j}}(j=1, \ldots, n)$. We shall consider the functions given by

$$
F(z)=z \prod_{j=1}^{n}\left(f_{j}(z) / z\right)^{a_{j}} .
$$

For a fixed $M>0$ denote by $\mathfrak{F}(M)$ the class of functions of the mentioned type for which

$$
\sum_{j=1}^{n}\left(1-\alpha_{j}\right)\left|a_{j}\right| \leq M
$$

THEOREM 1. The radius of starlikeness of the class $\mathfrak{F}(M)$ is $R^{*}=$ $1 /(2 M+1)$. The result is sharp for the function

$$
F(z)=z(1-z)^{2 M} .
$$

Proof. Fix $m \in(0, M]$ and $a \in[-m, m]$. Consider the subclass

$$
\mathfrak{F}_{a}^{(m)}=\left\{F \in \mathfrak{F}(M): \sum_{j=1}^{n}\left(1-\alpha_{j}\right) \operatorname{Re} a_{j}=a\right\} .
$$

By the analytic definition of starlikeness the radius $r^{*}$ of starlikeness of the class $\mathfrak{F}_{a}^{(m)}$ is the largest number $r \in(0,1)$ for which

$$
\min _{|z|=r} \operatorname{Re} \frac{z F^{\prime}(z)}{F(z)} \geq 0, \quad F \in \mathfrak{F}_{a}^{(m)} .
$$

For every $r \in(0,1)$ we have

$$
\begin{aligned}
\min _{|z|=r} \operatorname{Re} \frac{z F^{\prime}(z)}{F(z)} & =1+\min _{|z|=r}\left\{\sum_{j=1}^{n} \operatorname{Re}\left[a_{j}\left(\frac{z f_{j}^{\prime}(z)}{f_{j}(z)}-1\right)\right]\right\} \\
& \geq 1+\sum_{j=1}^{n}\left\{\min _{|z|=r} \operatorname{Re}\left[a_{j}\left(\frac{z f_{j}^{\prime}(z)}{f_{j}(z)}-1\right)\right]\right\} .
\end{aligned}
$$

Of course, if each real part in the last sum attains its minimal value at the same point, the inequality becomes equality.

From $f_{j} \in S^{*}\left(\alpha_{j}\right)$ it follows that

$$
\frac{z f_{j}^{\prime}(z)}{f_{j}(z)} \prec \frac{1-\left(2 \alpha_{j}-1\right) z}{1-z}, \quad|z|<1,
$$

hence

$$
\frac{z f_{j}^{\prime}(z)}{f_{j}(z)}-1 \prec \frac{2\left(1-\alpha_{j}\right) z}{1-z}, \quad|z|<1,
$$


By the subordination principle

$$
\left|\left(\frac{z f_{j}^{\prime}(z)}{f_{j}(z)}-1\right)-\frac{2\left(1-\alpha_{j}\right) r^{2}}{1-r^{2}}\right| \leq \frac{2\left(1-\alpha_{j}\right) r}{1-r^{2}}, \quad 0<|z|=r<1 .
$$

Hence

(1) $\min _{|z|=r} \operatorname{Re}\left\{a_{j}\left(\frac{z f_{j}^{\prime}(z)}{f_{j}(z)}-1\right)\right\} \geq \frac{2\left(1-\alpha_{j}\right) r \operatorname{Re} a_{j}}{1-r^{2}}-\frac{2\left(1-\alpha_{j}\right)\left|a_{j}\right| r}{1-r^{2}}$

with equality for the function $\tilde{f}(z)=z(1-z)^{-2\left(1-\alpha_{j}\right)}$. Consequently,

$$
\min _{|z|=r} \operatorname{Re} \frac{z F^{\prime}(z)}{F(z)} \geq \frac{(2 a-1) r^{2}-2 m r+1}{1-r^{2}}=P(r) .
$$

Since $m^{2}-2 a+1 \geq 0$ the zeros of the denominator of $P(r)$ are real. On the other hand, $P^{\prime}(r) \leq 0(r \neq \pm 1)$ and $P(0)=1$ yield the existence of a unique zero of $P(r)$ in the interval $(0,1)$. Irrespective of the sign of $2 a-1$ the zero in question is given by the expression

$$
\frac{m-\sqrt{m^{2}-2 a+1}}{2 a-1}=\frac{1}{m+\sqrt{m^{2}-2 a+1}} .
$$

Hence

Define

$$
r^{*}=\frac{1}{m+\sqrt{m^{2}-2 a+1}}
$$

$$
w_{j}=\frac{2\left(1-\alpha_{j}\right) r^{2}}{1-r^{2}}
$$

The transformation $W_{j}=a_{j} w_{j}$ maps the circle

$$
\left|w_{j}-\frac{2\left(1-\alpha_{j}\right) r^{2}}{1-r^{2}}\right|=\frac{2\left(1-\alpha_{j}\right) r}{1-r^{2}}
$$

onto the circle

$$
\left|W_{j}-\frac{2\left(1-\alpha_{j}\right) a_{j} r^{2}}{1-r^{2}}\right|=\frac{2\left(1-\alpha_{j}\right) a_{j} r}{1-r^{2}}
$$

whose leftmost point is the right-hand number in (1). To this point there corresponds a unique $w_{j}$ and a unique $z_{j}$ respectively. A sufficient condition for the coincidence of all $z_{j}$ is $\theta_{1}=\theta_{2}=\cdots=\theta_{n}=\theta_{0}$, i.e. when all circles are rotated through the same angle. Thus we arrive at the function

$$
\begin{array}{r}
\tilde{f}(z)=z \sum_{j=1}^{n}\left(\tilde{f}_{j}(z) / z\right)^{a_{j}}=z(1-z)^{-2 \sum_{j=1}^{n}\left(1-\alpha_{j}\right) a_{j}}=z(1-z)^{-2 m e^{i \theta_{0}}}, \\
\theta_{0}=\arccos (a / m),
\end{array}
$$

whose radius of starlikeness is exactly $r^{*}$. 
The expression $\left(m+\sqrt{m^{2}-2 a+1}\right)^{-1}$ is an increasing function of parameter $a$. Hence the radius of starlikeness of

$$
\bigcup_{-m \leq a \leq m} \mathfrak{F}_{a}^{(m)}
$$

is $1 /(2 m+1)$. Of course the extremal function becomes $\widetilde{f}(z)=z(1-z)^{2 m}$. Since $1 /(2 m+1)$ is a decreasing function of $m$ the radius of starlikeness of the class $\mathfrak{F}(M)$ is $1 /(2 M+1)$ with extremal function $\widetilde{F}(z)=z(1-z)^{2 M}$.

Consider functions of the type

$$
G(z)=\int_{0}^{z} \prod_{j=1}^{n}\left(f_{j}(u) / u\right)^{a_{j}} d u
$$

and denote by $\mathfrak{G}(M)$ the class of such functions for which $\sum_{j=1}^{n}\left(1-\alpha_{j}\right)\left|a_{j}\right| \leq$ $M$ for some fixed $M>0$.

THEOREM 2. The radius of convexity of the class $\mathfrak{G}(M)$ is

$$
R_{C}=\frac{1}{2 M+1} \text {. }
$$

The result is sharp for the function $\widetilde{G}(z)=\int_{0}^{z}(1-u)^{2 M} d u$.

Proof. Let $G \in \mathfrak{G}(M)$. Since $1+z G^{\prime \prime}(z) / G^{\prime}(z)=z F^{\prime}(z) / F(z)$ for some $F \in \mathfrak{F}(M)$, the proof is a repetition of the proof of Theorem 1 .

Special cases. Let $M>0$. For a fixed $A \in[-M, M]$ consider the class

$$
\mathfrak{F}_{A}=\left\{F \in \mathfrak{F}(M): A=\sum_{j=1}^{n}\left(1-\alpha_{j}\right) \operatorname{Re} a_{j} \leq M\right\} .
$$

Using the reasoning in the proof of Theorem 1 it is easily seen that the radius of starlikeness of $\mathfrak{F}_{A}$ is

$$
R_{A}^{*}=\frac{1}{M+\sqrt{M^{2}-2 A+1}}
$$

with extremal function $\widetilde{F}_{A}(z)=z(1-z)^{-2 M e^{i \theta_{0}}}, \theta_{0}=\arccos (A / M)$. For $\theta_{0}=0$, i.e. $A=M$, we obtain

$$
R_{M}^{*}=\frac{1}{M+|M-1|}= \begin{cases}1 & \text { if } 0<M \leq 1 \\ \frac{1}{2 M-1} & \text { if } M>1\end{cases}
$$

In short, the radius of starlikeness of $\mathfrak{F}_{M}$ is 1 if and only if $a_{j} \geq 0$ and $\sum_{j=1}^{n}\left(1-\alpha_{j}\right) a_{j} \leq 1$. Consequently this is the only case when we may speak of the order of starlikeness in the unit disc. 
Following Silverman [1] we find that

$$
\mathfrak{F}_{M} \subset S^{*}(\alpha), \quad \text { where } \quad \alpha=1-M=1-\sum_{j=1}^{n}\left(1-\alpha_{j}\right) a_{j} .
$$

Choosing $\alpha_{j}=\alpha$ or $a_{j}=a(j=1, \ldots, n)$ we obtain the results of Theorem 1 of [1] and Theorem 2 of [1] respectively.

Define

$$
\mathfrak{G}_{A}=\left\{G \in \mathfrak{G}(M): A=\sum_{j=1}^{n}\left(1-\alpha_{j}\right) \operatorname{Re} a_{j} \leq M\right\} .
$$

In the same way we obtain analogous results concerning $\mathfrak{G}_{A}$ and $\mathfrak{G}_{M}$.

Acknowledgements. The author is much obliged to Professor Zbigniew J. Jakubowski for his helpful remarks and advice during the preparation of the paper.

\section{References}

[1] H. Silverman, Products of starlike and convex functions, Ann. Univ. Mariae CurieSkłodowska 29(1975), 109-116.

[2] A. W. Goodman, Univalent Functions, Mariner, 1983.

INSTITUTE OF MATHEMATICS

BULGARIAN ACADEMY OF SCIENCES

P.O. BOX 373

1090 SOFIA, BULGARIA 